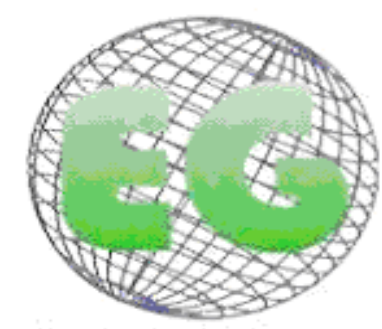

ISSN 1695-6141 N26

\title{
Las relaciones informales: un valor añadido en la gestión de personas
}

Informal relationships: an added value in human management

\author{
*Marqués Sánchez, P., " ${ }^{* *}$ Farrerons Noguera, L., ${ }^{* * *}$ Arias Ramos, N., "***Quiroga \\ Sánchez, E
}

*Profesora Titular de Administración de Servicios de Enfermería. Universidad de León (Campus de Ponferrada). E.mail: pilar.marques@unileon.es **Profesora Titular de Administración de Servicios de Enfermería. Universidad de Las Palmas de Gran Canaria. ****Enfermera del Hospital El Bierzo. Profesora Asociada de Prácticas Clínicas, Universidad de León. ${ }^{* * * *}$ Enfermera de la Junta de Castilla y León. Universidad de León.

Palabras clave: relaciones informales; organizaciones sanitarias; gestión enfermera, capital social. Keywords: Social Networks; performance; health organization; values; social capital.

\section{RESUMEN}

Las organizaciones sanitarias son consideradas por los expertos como una de las más complejas a nivel de gestión y política de personas. El hecho responde a la intensidad de las emociones vividas en el día a día tanto con el paciente como con los colegas.

Las experiencias vividas donde se combinan alegrías, tristezas y momentos estresantes generan en muchas organizaciones sanitarias un clima laboral inadecuado que repercute negativamente en el rendimiento de las organizaciones de salud. Este artículo pretende justificar teóricamente, la importancia de las relaciones sociales o informales que se establecen entre los trabajadores y cómo éstos pueden tejer un entramado por el que fluyan conocimiento, creatividad y valores que provoquen una cultura organizativa capaz de aumentar el rendimiento de la empresa sanitaria.

\section{ABSTRACT}

Health organizations are considered by experts how one of the most complexity about management and resources of human. The reason is a lot of strenght emotions lived every day so much with patients so with peers.

Lived experiences, where they are linked (combined), joy(felicity), sorrow and stress situations, can be the cause of wrong (bad) climate and so to impact (shaping) on the future performance of the health organizations. The objective of this paper is to argue the value of social relationship o informal relationship among workers and how those can knith (draw) a structure by the which, flow knowledge, creativity and values. Secondary to this, the organisationally culture is able to improve the performance of health enterprise. 


\section{INTRODUCCIÓN}

El pasado siglo se caracterizó por buscar nuevas fórmulas que apoyaran la competitividad de la empresa dotándola de estabilidad, expansión e innovación. El entorno cambiante tanto tecnológica como institucionalmente, enfrenta a empresarios y directivos hacia una incertidumbre donde las relaciones laborales, sociales e informales pueden convertirse en el activo más importante para dotar de sostenibilidad a la organización. (1) (2)

En este sentido, las políticas sanitarias también están buscando nuevos modelos de culturas, lenguajes y fórmulas que despierten en los médicos y en las enfermeras su compromiso con la organización y con su profesión. Parece que ésta podría ser la clave para dotar de sostenibilidad a nuestro sistema sanitario, y así lo avalan algunos estudios que hacen un llamamiento al aumento de responsabilización de todos los agentes del sistema, es decir, de pacientes, profesionales y gestores ${ }^{(3)}$.

Sin embargo, somos conscientes de que la carencia de incentivos ${ }^{(4)}$ y de motivación en el personal $^{(5)}$ ocasionan un gran número de actuaciones ineficientes que impactan directamente en el usuario. En palabras de Repullo( ${ }^{(6)}$, "los sanitarios deben apropiarse en positivo del SNS para defenderlo de todos los que por acción o por omisión lo ponen en peligro". Por tanto, para valorar este "empoderamiento", primero se debe identificar a un profesional como algo más que un actor con un rol aislado. No tendría mucho sentido hablar de buenos o malos profesionales únicamente en función, por ejemplo, de si administran toda la medicación en las horas pautadas, o de si tienen los registros bien cumplimentados o de si han logrado disminuir su gasto farmacéutico, entre otros. El rendimiento de un profesional sanitario ha de ser medido en función de todos los recursos que pone a disposición de su entorno laboral: las ideas, la colaboración, la información, el entusiasmo, la predisposición, etc. Por tanto hablamos de los trabajadores como actores dentro de un contexto social, donde si no te da tiempo a dar la medicación pides ayuda y eso es eficacia, donde a la hora de realizar un procedimiento que desconoces solicitas información porque así te encuentras más satisfecho, y donde si tienes una buena idea la compartes y la transfieres porque eres consciente que de otra forma no llegará a materializarse.

Hablamos, pues, de una forma de interactuar que no es más que las relaciones y conexiones que establecemos informalmente, presente en nuestras organizaciones, pero invisible a la hora de pensar en cómo optimizarla. Partiendo de esta premisa, surge la motivación del presente trabajo, que se fundamenta en una justificación teórica, a partir de una extensa revisión bibliográfica, sobre la relevancia del contexto social organizativo en el ámbito sanitario.

En esta línea aparecen numerosas investigaciones que estudian las distintas conexiones dentro de una organización, a nivel vertical y horizontal, y los comportamientos de los profesionales en el grupo de trabajo. ${ }^{(7)}$ Actualmente, las tendencias más innovadoras apuestan por conjugar la diversidad y la complejidad de los recursos humanos. ${ }^{(8) .}$ Estas contribuciones tienen, hasta ahora, poco "eco" en las organizaciones sanitarias, dónde las relaciones sólo son traducidas en una estructura formal y jerárquica. Sin embargo, nuestra propuesta no pretende anular las relaciones formales sino que intenta contemplar la arquitectura organizativa conjuntamente con el espacio de relaciones sociales, tal y como apunta el modelo de Nadler y Tushman. ${ }^{(9)}$

Por tanto, para conocer realmente hasta dónde las relaciones informales podrían aportar un valor añadido o no, a nuestro sistema, se procedió a la revisión de la literatura en dos ejes fundamentales para dar soporte a todo el marco teórico: Definir el constructo de relaciones 
informales junto con aquellas teorías que le den soporte como concepto teórico y enumerar y describir los contenidos principales que fluyen por sus conexiones. Para finalizar, se presentarán unas conclusiones con el objeto de poder aportar futuras reflexiones, especialmente para la práctica de la gestión enfermera.

\section{OBJETIVOS}

La revisión bibliográfica del presente artículo pretende conceptualizar las relaciones informales dentro de las organizaciones, con el objeto de hacerlo extensible y aplicable al contexto sanitario y, de forma más concreta, a la gestión de los recursos humanos en enfermería. Para materializar dicho fin, se han enunciado los siguientes objetivos:

- Conceptualizar las relaciones informales, de forma que seamos capaces de identificarlas en nuestros entornos de trabajo.

- Conocer aquellos marcos teóricos más relevantes que están próximos a las relaciones informales.

- Explorar qué contenidos fluyen a través de las conexiones informales y qué pueden aportar a la gestión enfermera.

\section{MARCO CONCEPTUAL: CONCEPTO Y TEORÍAS RELACIONADAS}

\section{Concepto de relaciones informales}

No es fácil encontrar el constructo de relaciones informales, más bien ha sido una evolución desde el marco de la comunicación para hacer referencia a las interacciones entre las personas en un contexto de cercanía, de amistad o de sintonía, rompiendo las barreras estructurales organizativas. Pero ciertamente, en la gestión enfermera y en el liderazgo de los mandos intermedios, siempre han sido reconocidas cómo útiles y necesarias. Es decir, cuando un trabajador comparte inquietudes laborales con otro compañero, bien del mismo departamento, de otro, con una supervisora, con un representante sindical, tomando un café en el office, o dando un paseo por la calle, estamos compartiendo una información y construyendo un conocimiento que, en la mayoría de las ocasiones, se pierde. Obviamente, una enfermera-líder debiera captarlo y potenciar estas acciones con el objeto de conseguir no sólo una mejora de la eficacia individual sino también colectiva ${ }^{(10)}$. Por esta razón, el gestor debe tener presente el potencial de las relaciones informales, adiestrarse en cómo se forman, y lo que conllevan.

El término de las relaciones sociales tenemos que contextualizarlo dentro de teorías como el Capital Social, la Teoría Institucional, el Intercambio Social y la Psicología Evolutiva, entre otras. Aquí confluyen numerosas disciplinas como las matemáticas, sociología, psicología, economía, antropología, etc., estudiando las relaciones informales como una estructura social. En ésta, un actor puede ser desde un individuo, hasta un departamento, una organización o una institución, según el nivel micro o macro al que hagamos referencia. Las relaciones son traducidas en conexiones entre diadas, triadas, unidireccionales, direccionales, etc., tejiendo una estructura rica en recursos. Así, por ejemplo, dos enfermeras que se coordinan perfectamente en el trabajo (diada y relación bidireccional), dos enfermeras y un supervisor que se transfieren todo tipo de información sobre los aspectos de mejora de su procesos (triada), o un médico que comunica todas las nuevas pautas terapéuticas a la enfermera pero la enfermera no le traslada las alteraciones que va observando en el paciente (información unidireccional). Por otra parte, estas conexiones se pueden establecer entre dos enfermeras de distintos servicios que se comunican sobre 
cómo aumentar la adherencia al tratamiento de sus pacientes, o entre los profesionales de un hospital que acuden a una reunión científica y establecen interacciones con otros colegas, las cuales revertirán positivamente en su trabajo diario.

Con respecto a estas estructuras informales, existe un gran número de contribuciones que también demuestran la importancia de la posición del individuo en el entramado de las relaciones, del comportamiento que adquiere por contagio, de las oportunidades de las conexiones, etc. Algunas de las más relevantes a nivel teórico son:

- Homans ${ }^{(11)}$ afirmó que los comportamientos emanan del contacto directo de unos actores con otros y eso genera actitudes sociales.

- Emerson ${ }^{(12)}$ presentó el modelo de comportamiento de acción individual a nivel macro. Para este autor, un actor es concebido como un punto donde conectan muchos intercambios de relaciones, formales e informales.

- Burt ${ }^{(13)}$ defendió que los intereses de un actor son el resultado de su posición dentro de la estructura social y marca la importancia que tiene la interacción social para conseguir sus metas.

- Wellman ${ }^{(14)}$ analizó las conexiones entre actores para conocer el comportamiento social y el intercambio social que se genera a través de la estructura social.

Estos autores han llevado sus investigaciones desde el campo sociodemográfico con estudios sobre vecindarios, al entorno de la empresa a finales del siglo pasado. Sus planteamientos parecen útiles para extrapolarlos al campo de la gestión enfermera, donde los aspectos, por ejemplo, de contagio social serían muy necesarios conocerlos en profundidad. Es decir, ¿pueden determinadas estructuras de red provocar que las enfermeras, médicos, y auxiliares se ayuden? o ¿algunos patrones de relaciones informales son responsables de una difusión de conflictos y rumores que no benefician al usuario ni al clima psicológico del equipo?.

Para dar respuestas a estas y a otras preguntas es necesario conocer algunos marcos teóricos que aborden estas cuestiones y profundizar en sus principales ejes que explícitamente demuestren la existencia y relevancia de las conexiones informales. Las teorías más relacionadas con estas estructuras sociales y que a continuación se exponen brevemente son: Teoría del Intercambio Social, Capital Social, Psicología Evolutiva y Teoría Institucional.

\section{Teoría del Intercambio Social}

La Teoría del Intercambio Social explica las dinámicas que se establecen entre los individuos, de forma que un actor voluntariamente facilita un beneficio a otro y de alguna forma espera reciprocidad para obtener también algún beneficio de esta relación ${ }^{(15)}$. Para este marco conceptual lo relevante es la estructura social que se genera a partir del intercambio de relaciones sociales y que permite la permuta de información, entre otros recursos. Así, Homans ${ }^{(16)}$ subraya la importancia del comportamiento que emana de los contactos entre los individuos y la repercusión que estos contactos puede tener dentro de cualquier estructura social.

Los investigadores aseguran que la conducta social, como cualquier otra conducta operante, puede ser fácilmente predecible si son analizadas sus consecuencias, de las cuales pueden ser extraídas las causas o los estímulos de dichos comportamientos. De esta forma, se podrían generar, suprimir o mantener comportamientos sociales actuando sobre las relaciones, las conductas particulares o las condiciones ambientales. Según Béjar, ${ }^{(17)}$ las conductas sociales son generadas por conductas pasionales y emocionales individuales; 
afirma que son las ideas y las pasiones, y no la mecánica de las leyes, lo que impulsa los asuntos humanos y hace referencia a la importancia del entusiasmo defendido por Kant como una participación afectiva que tendía a unir a los hombres hacia lo ideal y lo puramente moral.

Côté ${ }^{(18)}$ pone de relieve en una de sus investigaciones, que las personas que expresan sus emociones más agradables y positivas afrontan su trabajo invirtiendo menos esfuerzo y sufriendo menos estrés que las que expresan emociones más negativas.

La Teoría del Intercambio Social puede ser la base para defender cómo las conexiones ente las personas pueden convertirse en un valor añadido y diferencial para cualquier organización y cualquier equipo de trabajo, incluyendo los sanitarios. Es necesario, por tanto, que el intercambio de información y aspectos colaborativos se materialicen y se conviertan en una dinámica habitual. De esta forma, por ejemplo, las comisiones de calidad, investigación o docencia de un hospital, son espacios de intercambio muy productivos para la organización y que revierten determinado prestigio social a los profesionales. Otra situación en la cual veríamos aplicada esta teoría en nuestro ámbito sanitario sería con la presencia de un líder que propicie un entorno distendido y agradable para los trabajadores. Se ha demostrado que el optimismo y la afectividad entre los profesionales, ayudan a superar momentos de crisis e incluso lubrican las situaciones más conflictivas.

Los gestores enfermeros tienen, en esta teoría, un recurso para su práctica diaria. Con el intercambio social se consiguen recursos que realmente revierten motivación y resultados más óptimos, aspectos de vital importancia para cualquier organización.

\section{Capital Social}

El capital social es considerado como un marco conceptual clave en el campo organizativo. Define el conjunto de normas, relaciones e incluso organizaciones, a través de las cuales son alcanzados los recursos y las oportunidades que ayudan a tomar decisiones y a gestionar el trabajo eficazmente ${ }^{(19)}$.

El término capital social surge de los primeros estudios sobre comunidades de vecinos realizados para investigar cómo las relaciones, la cooperación y el desarrollo de la confianza facilitaban la supervivencia y el funcionamiento del vecindario en las ciudades. Numerosos autores $^{(20)(21)}$ lo han definido en función de la riqueza que generan las relaciones sociales a la organización, sin haber un gran consenso en cuanto a su conceptualización. El concepto estudia quién alcanza a quién ${ }^{(22)}$, qué tipo de relaciones tienen los actores entre ellos y cómo influyen en su comportamiento ${ }^{(23)}$, y qué recursos pueden ser compartidos, como por ejemplo, propósitos, códigos, mitos, símbolos, etc.

Podemos afirmar, pues, que el capital social forma parte de las relaciones y que puede crear ventaja competitiva tanto a nivel individual como social ya que los trabajadores que están mejor conectados lograrán mejores resultados. ${ }^{(24)}$ Por lo tanto, este concepto tiene una importancia respecto a la localización en la estructura organizativa que exige a los directivos un conocimiento estrecho de las actitudes y habilidades de los actores que están bajo su responsabilidad. De esta forma, trabajadores con más afinidades tenderán a conectar más directamente lo que mejorará la comunicación, ${ }^{(25)}$ y la confianza ${ }^{(21)}$ que redundará en un rendimiento más exitoso.

La aplicación del Capital Social en el ámbito sanitario la podemos observar, por ejemplo, en las formas de promoción para participar en órganos de representación sindical, asesores o 
incluso para acceder a un puesto directivo. En todas estas situaciones, el actor va tejiendo su propia de red de relaciones donde el poder de cada contacto es analizado con precaución. Al hablar de poder en las estructuras sociales, hacemos referencias al grado de prestigio que un nodo (compañero) tiene en un determinado campo de actuación. Así, si yo quiero tener información sobre nuevas oportunidades, escogeré cuidadosamente mi primer contacto, el cual me revierta información rápidamente y de forma fiable. En caso de acceder indirectamente a los contactos de mis contactos, el grado de confianza disminuye.

En el caso de una enfermera que pretende alcanzar un puesto en la Dirección de Enfermería, con la cual no tiene contacto cercano con ella, deberá "tejer" toda una estrategia para que de ella lleguen referencias a la dirección, y que de la dirección le llegue información sobre qué perfil de supervisora o colaboradora necesitan.

Por tanto, el Capital Social nos muestra la importancia de nuestra posición en una red, puesto que dependiendo de esa localización tenemos más o menos facilidad para alcanzar a otros contactos, lo que conlleva posibilidades y, por ello, aspectos que nos importan a nivel laboral.

\section{Psicología Evolutiva}

La Psicología Evolutiva ha tenido como meta el estudio del ciclo vital para mejorar tanto en la calidad de vida personal como a nivel de la producción en entornos organizativos. Su conocimiento facilita la autoevaluación, la mejora de uno mismo ${ }^{(26)}$ y ejercer el autocontrol. (27)

La teoría explica cómo el desarrollo del ser humano a veces es cíclico, con replanteamientos de roles en las distintas etapas de la vida por lo que las mismas fases pueden ser experimentadas por distintos individuos, a pesar de sus diferencias individuales y culturales (27) y en momentos similares o diferentes.

En este sentido, y aunque si bien es cierto que no se han encontrado trabajos en la literatura que relacionen directamente esta teoría con el marco de las relaciones informales, las aportaciones que hace sobre cómo las personas se enfrentan a los cambios personales y sociales se ha considerado de especial relevancia para este artículo. La principal contribución se deriva de la forma en que cada individuo hace frente a dichos cambios, siendo ese comportamiento una consecuencia de "su propia flexibilidad y capacidad de adaptación, de su motivación para lograrlo y de su disposición para asumir su responsabilidad personap"(27).

Así, según la Psicología Evolutiva, lo más eficaz sería que la persona cambiara porque ella misma quiere afrontar los cambios, aunque para ello necesite del apoyo de sus compañeros o de sus responsables jerárquicos. Esta reflexión lleva a considerar qué aspectos, cognitivos o emocionales, se deberían de valorar en los miembros de una organización con el fin de poder afrontar con más eficacia los cambios sociales organizativos, aspecto clave en esta disertación. Así, algunas cuestiones al respecto podrían ser: el estudio sobre cómo el cambio de los ciclos vitales individuales de las enfermeras afecta al grupo de trabajo, el interés por conocer los aspectos relacionales que puedan moderar los cambios del ciclo vital de los individuos en el contexto organizativo, o cómo el funcionamiento de los ciclos vitales repercute en las capacidades de liderazgo de una supervisora.

Siguiendo con este argumento, la Psicología Evolutiva puede ayudar a comprender mejor los comportamientos de las enfermeras, médicos y auxiliares dentro de una estructura social, 
debido a que explica una serie de patrones de comportamiento que tienen lugar en las diferentes etapas de la vida. Es cierto que no se han encontrado estudios que demuestren esta relación y que tampoco existe demasiado consenso a la hora de valorar la parte más emocional de los trabajadores con respecto a su rendimiento. Sin embargo, algunos autores defienden que las personas actúan por mecanismos instintivos para resolver problemas y explican cómo estos mecanismos responden, tanto a las condiciones de la propia naturaleza humana, como al contexto que rodea al actor ${ }^{(28)}$.

Por lo tanto, todos estos aspectos son relevantes a la hora de organizar las plantillas de personal en cualquier centro sanitario. Habrá que plantearse si es más interesante reunir en un equipo personal novel con senior, o si a la hora de abrir un nuevo servicio se necesita un grupo de profesionales que puedan dar un mayor compromiso, aunque sea de forma temporal, debido a que sus cargas familiares se lo permiten, etc.

En un esfuerzo integrador, en la siguiente tabla se han resumido las aportaciones de Gould y Levinson ${ }^{(29)(30)}$. Este contenido puede ser muy útil para la enfermera gestora a la hora de reflexionar sobre su movilidad interna, por ejemplo. Tiene aquí, una herramienta que responde a una contribución de la literatura seguida por numerosas investigaciones.

\begin{tabular}{||l|l||}
\hline \multicolumn{2}{||c||}{ Propuesta integradora del ciclo vital conjugando a Gould y Levinson } \\
\hline \hline Años & Descripción \\
\hline $18-23$ años & Mucho impulso y poca orientación. \\
\hline $23-30$ años & Participación en el mundo a dulto. Se fijan metas. \\
\hline $30-35$ años & Se autocuestionan roles. Etapa de vida en pareja estable y nacimiento de hijos. \\
\hline $35-40$ años & $\begin{array}{l}\text { Asentamiento para conseguirlas metas. Conflictos entre el crecimiento } \\
\text { profesionaly crecimiento personal. }\end{array}$ \\
\hline $40-50$ años & Reevaluación del tiempo pasado, reorientación de las metas. \\
\hline $50-60$ años & Etapa estable y dulce si está satisfecho con sus etapas anteriores. \\
\hline
\end{tabular}

A modo de conclusión, la Psicología Evolutiva nos está indicando que las relaciones informales también cambian a la vez que lo hacen los ciclos vitales de los profesionales de un equipo de salud y, además, que es relativamente predecible puesto que se generalizan una serie de comportamientos en unas determinadas franjas de edad.

\section{Teoría Institucional}

Las organizaciones tienen una cierta "permeabilidad", metafóricamente hablando, a través de sus barreras formales. Esto quiere decir, que se asume la presencia de un flujo de conexiones establecido entre sus miembros y otras instituciones. Parece ser debido a que el entorno que rodea a la empresa puede demandar ciertos productos o servicios, o ser fuente de amenazas y oportunidades, aspectos todos ellos que han de ser considerados para su propio beneficio. La Teoría Institucional recoge esta idea, por lo que da una importancia especial a las redes sociales que se generan entre la organización y su entorno.

La Teoría Institucional ha experimentado una evolución tan importante que se habla del viejo institucionalismo y del nuevo institucionalismo o Neoinstitucionalismo ${ }^{(31)}$. El primero, se centra en la organización desde una perspectiva más del individuo mientras que el segundo, 
hace alusión al campo organizativo como conjunto de organizaciones e instituciones que definen un contexto y que puede influir en la estructura social ${ }^{(32)}$.

Esta teoría otorga gran importancia a las relaciones sociales fuera de la organización puesto que mediante estos vínculos, se pueden lograr no sólo nuevos éxitos sino también conocer otras posibilidades que le apoyen en su consolidación. Estos objetivos se consiguen reflexionando también sobre las metas que tiene el entorno, y reconociendo que esas metas influyen en el comportamiento de las organizaciones ${ }^{(33)}$. Para conseguir este equilibrio, la organización ha de alcanzar un apoyo social, el cual se logra cuando se adapta a los procesos, las normas y a los valores externos ${ }^{(34)}$.

En este sentido, se podría pensar en cómo optimizar las relaciones sociales que algunas enfermeras o directivos mantienen con grupos de interés del exterior. Sin embargo, puede que el individuo no esté motivado para desarrollar esta estrategia si no obtiene un reconocimiento social. Burt ${ }^{(13)}$ afirma que los individuos se motivan según la percepción que tienen de su reconocimiento dentro de una red social. Por esta razón, habría que pensar en algún tipo de recompensa o prestigio social para aquellos profesionales sanitarios que consiguieran trabajar con el entorno y no perdieran su lealtad, en cuanto a que todos los beneficios conseguidos revirtieran en su empresa. Romney y Faust ${ }^{(35)}$ y Freeman $^{(36)}$ aseguran que las personas recuerdan con más exactitud y durante más tiempo aquellos acontecimientos en los que han tenido lugar interacciones sociales, que aquellos otros en los que actúan aisladamente. Parece, por tanto, útil diseñar estructuras informales de colaboración interorganizativas que sirvan de transferencia de ideas, conocimiento y, a la vez de autoestima para los profesionales.

En esencia, y paralelamente a estas aportaciones, se podría concluir que los trabajadores competentes para obtener beneficios desde el exterior hacia la organización, deberían estar inmersos en una estructura social que les reconozca y les apoye socialmente ${ }^{(13)}$.

Por otra parte, las organizaciones desarrollan su actividad económica dentro de un contexto social, hasta el punto que el proceso de toma de decisiones frecuentemente responde a las presiones derivadas de ese contexto. Este fenómeno se denomina inmersión institucional y es la consecuencia de las interconexiones entre una población y su contexto institucional.

También las organizaciones sanitarias, hospitales y centros ${ }^{(32)(37)}$ de salud, están influenciadas por políticas sanitarias locales, regionales y de gobierno central. La forma en la que sus directivos trabajen con estos agentes institucionales, revertirá con ventajas o inconvenientes en sus organizaciones.

A modo de resumen y como puede observarse, la investigación en el campo de las relaciones informales es tan extensa y con tantas posibilidades que la convierten en un campo emergente para las organizaciones. Nuestro entorno sanitario es un "lecho" perfecto para este nuevo diseño organizativo. La razón es el diverso perfil de actores, de los servicios prestados y de un entorno laboral impregnado de muchas sensibilidades, emociones, valores y sentimientos que se pueden ordenar y optimizar, tal y como lo defienden las teorías anteriormente expuestas.

\section{LOS CONTENIDOS DE LAS RELACIONES INFORMALES}

En las redes informales, existen canales de comunicación a través de los cuales fluyen contenidos que no pueden ser estudiados aisladamente sino que se interrelacionan, se expanden, se contraen y tienen movimiento predecible. Hablamos de informaciones, rumores, motivaciones, etc., que se traducen en conocimiento, afinidades (homofilia) y 
valores. En los siguientes epígrafes se expondrán brevemente estos contenidos de las relaciones informales.

\section{Conocimiento}

La naturaleza del conocimiento es en sí, intangible e inobservable pero capaz de materializarse en resultados, actividades e innovación. La singularidad y exclusividad que una organización tenga respecto a su conocimiento, jugarán un papel fundamental en su habilidad de competir ${ }^{(38)}$. Por lo tanto, los directivos han de gestionarlo mediante sistemas de control $^{(39)}$ que incluyan, los procedimientos y las rutinas formales e informales, los procesos de coordinación, y el control de las normas y las buenas prácticas, dentro de la misma.

Una de las líneas que más se está investigando sobre la gestión del conocimiento a través de las relaciones informales es la emergencia de la innovación. En toda organización existen pequeñas áreas donde surge la creatividad y la generación de nuevas y buenas ideas. La pregunta es cómo estas pequeñas estructuras pueden emerger y llegar a integrarse en la organización. Para Anand, Gardner y Morris ${ }^{(40),}$ habría que combinar cuatro aspectos: reconocer a la organización como un ente social, potenciar el conocimiento experto, ser un territorio dotado de fortaleza, y contar con el apoyo organizativo. Finalmente esa pequeña estructura o comunidad de trabajadores lograrán optimizar su conocimiento repercutiendo en un mayor rendimiento.

En esta línea se comienza a hablar de una organización como una constelación de comunidades para lo cual nuestra visión directiva y de gestión tendría que someterse a un gran cambio estructural y psicológico, pero que de alguna forma ya está en marcha. Estamos hablando de todas las veces en las que los profesionales de enfermería acuden a los congresos, comparten con sus colegas conocimientos y regresan a su servicio entusiasmados de haber hecho una propuesta atractiva en un foro científico, o de traerse una propuesta innovadora que dará solución a un problema recurrente en su servicio.

Siguiendo con los ejemplos, es frecuente que los directivos acudan a comisiones de su comunidad autónoma, donde en el mismo sentido se comparten ideas, problemas y experiencias. En toda esta transferencia no podemos olvidar, a los profesionales que están inmersos en las redes digitales y que comparten conocimiento con otros muchos que tienen un perfil similar a ellos. Realmente pueden contribuir con nuevas ideas a su equipo de trabajo, puesto que superan una de las barreras por las cuales en los centros sanitarios es difícil que surja la creatividad y la innovación: la causa es la información redundante. Así, es complejo que surjan nuevas propuestas cuando estás siempre en el mismo entorno, hablando y experimentando lo mismo. La creatividad aparece cuando tus contactos tienen orígenes distintos. Por tal cuestión, las relaciones a distancia y esporádicas facilitan nuevo conocimiento.

Todas estas relaciones y sus contenidos, no los tenemos presentes a la hora de explotarlos. Efectivamente forman una comunidad virtual rica en recursos y a tener en cuenta por las enfermeras gestoras para explotarlos en favor de la organización y de los propios profesionales.

\section{Homofilia}

Este concepto hace referencia a la similitud entre las características personales de los miembros de una comunidad, grupo u organización. Aspectos como los años de experiencia, edad, género, estamento y raza, pueden ayudar a mantener relaciones recíprocas. (41) 
Algunos trabajos asocian los aspectos de homofília con el número de contactos, ${ }^{(42)}$ así sean cual sean los aspectos de semejanza siempre serán más centrales y con más prestigio en la estructura social, los grupos mayoritarios que las minorías. ${ }^{(43)}$

Existen investigaciones que aportan evidencia sobre la relación existente entre la homofilia y el rendimiento individual o grupal. Este tipo de contactos que responden a las similitudes entre individuos, pueden repercutir positivamente en la organización debido a que: "facilitan la transmisión del conocimiento, simplifican la coordinación y evitan conflictos potenciales"(44). De acuerdo con esta idea, cabe pensar que las personas interactuarán y colaborarán más si tienen algún punto en común.

Precisamente, algunos estudios sobre relaciones han refrendado cómo las similitudes entre los individuos repercuten en un sentimiento de identidad y por tanto de compromiso hacia la organización ${ }^{(45)}$. Por tanto, la cuestión de la homofilia puede facilitar la ayuda y la colaboración, más entre unos trabajadores que entre otros, debido a creencias o aspectos culturales, como por ejemplo: mujer-mujer, enfermera-enfermera, veterano-veterano, blancoblanco, etc. Según estas contribuciones, la afinidad que más se mantiene en el tiempo es la experiencia o la veteranía en la organización, las demás aparecen con más frecuencia en un principio, hasta que el trabajador se integra en el equipo.

A nivel de gestión enfermera, este dato resulta de lo más interesante. Los responsables de las políticas de personal tendrán que pensar en una sabia combinación de las plantillas para optimizar sus relaciones. Por otra parte, no cabe duda, que en nuestros entornos existen claras afinidades entre los estamentos: los médicos se relacionan más con los médicos, enfermeras con enfermeras, auxiliares con auxiliares, etc. Esto no tendría mayor relevancia a no ser que veamos que los equipos no funcionan como tales. Es decir, un equipo de trabajo formal, como el servicio de medicina interna, puede realmente que no funcione como un todo, y que existan otros muchos sub-equipos que fraccionan la eficacia de resultados de un proceso asistencial. Por lo tanto, los responsables deben incorporar dinámicas de trabajo para que la interrelación entre los estamentos sea una costumbre.

\section{Valores}

Son numerosas las investigaciones que aportan datos sobre la importancia de diseñar un armazón social para la transmisión de valores. La propagación de los mismos genera ambientes que también se pueden optimizar. Hay que buscar valores que motiven individualmente y se alineen con el rendimiento organizativo ${ }^{(46)}$, pues, como sabemos, los comportamientos organizativos son el producto de las ideas, valores y creencias presentes en el entorno laboral.

Hasta ahora, los valores más estudiados han sido la justicia, la confianza, la creatividad y la innovación. (46) (47) (48) Todos ellos pueden, a la vez, estar relacionados con aspectos de homofilia y transmisión de conocimiento. Concretamente, los valores en nuestra profesión han sido analizados por un grupo de expertos y materializados en el documento denominado "Manifiesto de Espinoso de Compludo", delimitando como los más importantes el compromiso social, la seguridad, la armonía y la confianza. ${ }^{(49)}$

En esta línea, las últimas investigaciones sobre valores en las organizaciones, en general, hablan de valores muy similares al Manifiesto, como son la "buena voluntad", la "ciudadanía", el "compartir emociones" y la "afectividad". (50) Podemos establecer un símil entre las relaciones informales y un contagio de emociones y actitudes favorables, por lo tanto habrá que prever determinadas "vacunas" y "vitaminas" para cuando la organización se 
encuentre con una salud disminuida. En este sentido, las enfermeras gestoras han de captar quiénes de sus trabajadores son capaces de trasladar determinados valores. Por ejemplo, el que transfiere más afectividad, bondad y alegría, el cual será reconocido como el compañero entrañable y capaz de superar los momentos más tensos. Otro ejemplo, quien transmita seguridad, probablemente un profesional bien formado en conocimientos y con mucha destreza en las técnicas más complejas, referente, por tanto, en aquellas situaciones que impliquen una respuesta rápida y segura.

Resumiendo, las relaciones informales transfieren valores que pueden ser materializados en acciones útiles para los equipos y las organizaciones. Los directivos y los profesionales deben ser conscientes de tal realidad y alimentar este tipo de relaciones.

\section{CONCLUSIONES}

Brevemente hemos expuesto sólo una mínima parte del potencial que pueden generar las relaciones informales en nuestras organizaciones. El planteamiento que surge a partir de aquí es poder observar las estructuras organizativas con un ángulo más, añadido a la horizontalidad y a la verticalidad que conocemos. El carácter tridimensional nos exigirá un gran esfuerzo, especialmente cultural. El objetivo resultará todo un reto para los departamentos de recursos humanos, pero gracias a esta nueva óptica se podrán posicionar actores dentro de una red de relaciones según sus habilidades de conexión, experiencia, mediación, carácter emprendedor, etc.

El artículo demuestra teóricamente que las relaciones informales existen y, además, que otras teorías así lo avalan. Por lo tanto, es un dato más a tener en cuenta por las enfermeras gestoras que han de contemplar qué tipo de relaciones informales están presentes en sus equipos, cuándo y dónde se potencian y qué momentos las favorecen. En el mismo sentido, debemos tener presentes los recursos que fluyen a través de esas conexiones y que rompen la estructura jerárquica y formal de cualquier centro sanitario. La cuestión clave está en identificar cuáles son los contenidos, si son positivos o negativos para la organización y para los profesionales, y qué actores son los impulsores de los mismos. A partir de todo este análisis se pueden potenciar costumbres para propagar los recursos más óptimos. Entre algunos centros sanitarios existen comisiones intraorganizativas que trabajan en tópicos concretos como la seguridad del paciente, la adherencia al tratamiento, etc. Estos casos ya son un buen ejemplo de este tipo de transmisiones. En otras situaciones, los líderes directivos buscan estrategias en las que profesionales de distintos servicios tengan sesiones sobre temas de interés y, muchas veces, incluso en entornos externos a los laborales. Otras experiencias, están recogiendo antiguas costumbres sobre celebraciones institucionales donde se premia la fidelidad, el compromiso, la predisposición, etc.

El futuro para poder optimizar las relaciones informales en nuestras organizaciones sanitarias pasa por llevar a cabo un análisis más profundo y empírico que implica la aplicación metodológica del denominado Análisis de Redes Sociales, y que podrá convertirse en una herramienta más en la gestión enfermera. Debemos tener presente que nuestros centros son organizaciones abiertas que se adaptan a las necesidades sociales y al bienestar ${ }^{(51)}$, por lo tanto el reconocimiento de todas estas conexiones puede convertirse en un valor añadido y, por ello, ser de utilidad para avalar la sostenibilidad del sistema.

\section{REFERENCIAS}

1. BARNEY, J.B. (1991): "Firm resources and sustained competitive advantages". Journal of Management, 17, 99-120. 
2. BARNEY, J.B., WRIGHT, P. (1998): "On becoming a strategic partner. The role of human resources in gaining competitive advantage". Human Resource Management, 37 (1), 31-37.

3. BELTRAN, A.; FORN, R.; GARICANO, R., MARTINEZ, M.M. \& VAZQUEZ, P. (2009): Informe McKinsey: "Impulsar un cambio posible en el sistema sanitario".

www.cambioposible.es/sanidad

4. CASAS, J.; REPULLO, J.R. \& LORENZO, S. (2002): "Dimensiones y medición de la calidad de vida laboral en profesionales sanitarios". Revista de Administración Sanitaria, vol. VI, 23: 143-160.

5. GRAU, A.; SUÑER, R. \& GARCÍA, M.M. (2005): "Desgaste profesional en el personal sanitario y su relación con los factores personales y ambientales". Gac. Sanit. v.19,6, novdic.

6. REPULLO, J.R.. (2011): "Garantizar la calidad del Sistema Nacional de Salud en tiempo de crisis: sólo si nos comprometemos todos". Rev. Calid. Asist. 26 (1): 1-4.

7. KENNETH, A.F., JEFFREY, Y. (1998): "Linking action to social structure within a system: Social capital within and between subgroups". American Journal of Sociology, 104 (3), 642 645.

8. LENGNICK-HALL, C.A., SANDERS, M.M. (1997): "Designing effective learning systems for management education: student roles, requisite variety, and practicing what we teach". Academy of Management Journal, 40 (6), 1334-1368.

9. NADLER, D.A. y TUSHHMAN, L.M. (1997): Competing by design. The power of organizational architecture, New York, Oxford: Oxford University Press.

10.DE LIMA, L.; COELHO,S.; ADYLES, L.; BIOLCHI, T.; PIRES, DE., \& SCHUBERT, VM. (2011): "Influencia de los estilos de liderazgo del enfermero en las relaciones interpersonales del equipo de enfermería". Enfermería Global, vol. 10, no 22.

11. HOMANS, G.C. (1950): The Human Group. New York: Harcourt, Brace \& World. [REILLY DE FAYARD, M. (1977): El Grupo Humano. 5th edition. Buenos Aires: EUDEBA].

12.EMERSON, R.M. (1972): "Exchange theory part II: exchange rules and networks". In BERGER, J., ZELDITCH, M., ANDERSON, B., Editors. Sociological Theories in Progress, Boston: Houghton Mifflin, pp. 58-87. Cited by COOK, K.S., WHITMEYER, J.M. (1992): "Two approaches to social structure: exchange theory and network analysis". Annual Review of Sociology, 18, 109-127.

13.BURT, R.S. (1982): Toward a Structural Theory of Action: Network Models of Social Structure, Perceptions and Action. New York: Academic Press.

14. WELLMAN, B. (1983). "Network analysis: some basic principles". In: COLINS, R., Editor. "Sociological Theory", Jossey-Bass: San Francisco, pp. 155-200.

15. BLAU, P.M. (1964): Exchange and power in social life, New York: Wiley en WHITENER, E.M., BRODT, S.E., KORSGAARD, M.A. \& WERNER, J.M. (1998): "Managers as initiators of trust: an exchange relationship framework for understanding managerial trustworthy behavior", Academy or Management Review, 23 (3): 513-530.

16. HOMANS, G.C. (1964): "Bringing men back in", American Sociological Review, 29: 809818.

17.BEJAR, H. (1993): La Cultura del Yo. Pasiones Colectivas y Afectos Propios en la Teoría Social. Madrid: Alianza Editorial, S.A.

18. CÔTÉ, S. (2005): "A social interaction model of the effects of emotion regulation on work strain". Academy of Management Review, 30 (3), 509-530.

19.REQUENA, F. (2008): Redes Sociales y Sociedad Civil, Madrid: Centro de Investigaciones Sociológicas.

20.BOURDIEU, P.(1986) : The forms of capital. In Richardsonm, J.G. (Ed.). Handbook of theory and research for the sociology of education, New York: Greenwood en NAHAPIET \& GHOSHAL, S.(1998): "Social Capital, Intellectual Capital, and the organizational advantage", Academy of Management Review, 23(2): 242-266. 
21. COLEMAN, J.S. (1990) : Foundations of social theory, Cambridge, M.A. : Belknap Press of Harvard University Press en NAHAPIET, J.E. \& GHOHAL, S. (1998) : "Social Capital, Intellectual Capital, and the organizational advantage", Academy of Management Review, 23(2): 242-266.

22.BURT, R.S. (1992): Structural holes: The Social Structure of Competition, Cambridge; MA: Harvard University Press.

23. GRANOVETTER, M.S. (1992): "Problems of explanation in economic sociology" en NOHRIA. N. \& ECCLES R. Eccles, Networks and organizations: Structure, form and action, Boston: Harvard Business School Press

24.BURT, R.S.; HOGARTH, R.M., MICHAUD, C. (2000): "The social capital of French and American Managers". Organization Science, 11 (2), 123-147.

25. BAKER, W.E., IYER, AV. (1992). "Information networks and market behavior". Journal of Mathematical Sociology, 16 (4), 305-332.

26. HECKHAUSEN, J., KRUEGER, J. (1993): "Developmental expectations for the self and most other people: age grading in three functions of social comparison". Developmental Psychology, 29 (3), 539-548.

27. RICE P.F. (1997): Desarrollo Humano. Estudio del ciclo vital. JUÁREZ, N., Editor. México: Prentice-Hall Hispanoamericana, S.A.

28. GOLEMAN, D. (1996): Inteligencia emocional, Barcelona: Editorial Kairós, S.A.

29. GOULD, R.L. (1978): Transformation: Growth and change in adult life, New York: Simon \& Schuster, en RICE, F.P. (1997): Desarrollo Humano.Estudio del ciclo vital, Mexico: Prentice-Hall Hispanoamericana,S.A.

30. LEVINSON,D.J.(1978): "The midlife transition: A period in adult psychosocial development", Psychiatry, 40:99-112.

31. SELZNICK, P. (1996): "Institucionalism old and new", Administrative Science Quarterly, 41(2): 270-277.

32. DIMAGGIO, P.J. \& POWELL, W.W. (1983): "The iron cage revisted: Institutional isomorphism and collective rationality", American Sociological Reviews, 48: 147-160.

33. MEYER, J.W. \& ROWAN, B. (1977): "Institutionalized organizations: Formal Structure as myth and ceremony", American Journal of Sociology, 83:340-363.

34. MAZZA, C. \& ALVAREZ, J.L. (2000): " Haute couture and pret-â-porter: The popular press and the difusion of management practices", Organization Studies,21 (3): 567-588.

35. ROMNEY,A.K. \& FAUST, K. (1982): "Predicting the structure of a communications network from recalled data", Social Networks,4; 285-304.

36. FREEMAN, S. (1986): "Structural complexity and the recall of events". Paper presented at the Sixth Sunbelt Social Network Conference, Santa Barbara. Cited by KRACKHARDT, D. (1987): "Cognitive social structures". Social Networks, 9, 109-134.

37. DIMAGGIO, P.J. \& LOUCH, H. (1998): " Socially embedded consumer transactions: For what kinds of purchase do people most often use networks?. American Sociological Review, 63: 619-637 en BORGATTI, S.P. \& FOSTER, P.C. (2003): " The network paradigm in organizational research: A review and typology", Journal of Management, 29: 991-1013.

38. CONNER, K.R., LEVINTHAL, D.A. (1996): "Absorptive capacity: A new perspective on learning and innovation". Administrative Science Quarterly, 35 (1), 128-152.

39. TURNER, K.L., MAKHIJA, M.V. (2006): "The role of organizational controls in managing knowledge". Academy of Management Review, 31 (1), 197-217.

40. ANAND, N., GARDNER, H., MORRIS, T. (2007): "Knowledge-based innovation: emergence and embedding of new practice areas in management consulting firms". Academy of Management Journal, 50 (2), 406-428.

41. ABRAMS, L.C.; CROSS, R.; LESSER, E., LEVIN, D.Z. (2003): "Nurturing Interpersonal Trust for Knowledge-Intensive Work”. Academy of Management Executive, 17 (4), 64-95. 
42. MEHRA, A., KILDUFF, M., BRASS, D.J. (1998): "At the margins: a distinctiveness approach to the social identity and social networks of underrepresented groups". Academy of Management Journal, 41 (4), 441-452.

43. MICHAELSON, C. (2005): "Dialogue". Academy of Management Review, 30 (2), 235-238. 44.BORGATTI, S.P. \& FOSTER, P.C. (2003): "The network paradigm in organizational research: A review and typology", Journal of Management, 29 (6): 991-1013.

45. IBARRA, H.; KILDUFF, M. \& TSAI, W. (2005): "Zooming in and out: Connecting individuals and collectivities at the frontiers of organizational network research", Organization Science, 16 (4): 359-371.

46. JONI, S.N. (2004): "La geografía de la confianza". Harvard Deusto Business Review, 128, 60-67.

47.ROBERSON, Q., COLQUITT, J. (2005): "Shared and configural justice: a social network model of justice in teams". Academy of Management Review, 30 (3), 595-607.

48. INKPEN, A., TSANG, E. (2005): "Social capital, networks, and knowledge transfer". Academy of Management Review, 30 (1), 146-165.

49. MARQUES, P., MONPART, M.P., DIAZ, M.T., LEGARRA, S., MARTIN, A., NOGUES, C., GUILLEN, I., SILVEIRA, A., SILVEIRA, J., LOPEZ, J.J., ROMAGUERA, S., MERAYO, E., DUQUE, F.J., VAZQUEZ, E., ITURRIETA, A., GOMEZ, O., BELMONTE, T., IBARBUREN, R., LOPEZ, J., ARROYO, M.P., DURAN, M., FARRERONS, L., CALVO, M.D.,(2003):“Nursing Care: Management by values”. Hospital. Focus. 5 (3), 70.

50. BARON, R.A. (in press): "The role affect in the entrepreneurial process". Academy of Management.

51. LOPEZ MONTESINOS, M.J. (2009): "Consecuencias psicosociales del trabajo en el personal de enfermería como indicadores subjetivos de rendimiento desde el enfoque de la gestión de recursos humanos." Tesis doctoral. Repositorio Institucional DIGITUM. Base de datos TDR. Universidad de Murcia. ISBN: 978-84-692-9360-7. Depósito legal: MU-147-2010 En: http://www.tesisenred.net/TDR-0122110-124704/index_cs.html

\section{http://digitum.es}

ISSN 1695-6141 